\title{
PROKLA-Redaktion
}

\section{Editorial: Neue Waffen - neue Kriege?}

"Die Bilder von Frauen, die der Welt ihre lachenden Gesichter zeigten, von Männern, die ihre Bärte abrasierten, von Mädchen in der Schule, von Jungen, die in kurzen Hosen Fußball spielten: All das war zweifellos ein Schlag ins Gesicht linker Theorien über den amerikanischen Imperialismus..." - so schreibt einer der prominenten Unterzeichner des US-amerikanischen Manifestes für den Krieg gegen Terror, Michael Walzer, und belegt damit, wie selbst renommierte Sozialwissenschaftler sich derzeit wieder für die Idee vom "gerechten Krieg" erwärmen. Wer würde ernsthaft der gestürzten Taliban-Regierung eine Träne nachweinen, wer der afghanischen Bevölkerung nicht ihre lang entbehrten Freiheiten gönnen, selbst wenn sich diese - nach allem, was man weiß - auf Ausschnitte des Alltagslebens beschränken und möglicherweise nur für einige wenige gelten? Müssen aber solche Schnappschüsse damit einhergehen, dass viele der Fragen, die zumindest in den ersten Wochen des Krieges häufig gestellt wurden, nunmehr obsolet erscheinen? Damals wurde noch darüber diskutiert, dass die USA selbst es waren, die den politischen Aufstieg der Taliban mit Wafferlieferungen begünstigt hatten; dass das ursprüngliche Ziel des Krieges ein ganz anderes war, nämlich Bin Laden tot oder lebendig zu fangen; dass die als unterstützungswürdig geltende Nord-Allianz eine WarlordGruppierung darstellt, die den Taliban in ihren Methoden kaum nachstand; dass zwar über die finanziellen Kosten des
Krieges informiert wurde, bis heutc über die Zahl seiner zivilen Opfer aber Stillschweigen bewahrt wird. Solche Zusammenhänge werden mittlerweile nur noch vereinzelt thematisiert, doch selbst wenn vielen Kommentatoren Bushs alttestamentarisch inspirierte Rhetorik, die sich um Gott und um "das Böse“ in der Welt rankt, etwas bombastisch und überzogen scheint, so hat offenbar mittlerweile eine resignierte Gewöhnung an die neue Normalität stattgefunden: die Normalität militärischer Lösungen für die Probleme dieser Welt, die von westlichen (oder auch von östlichen) Regierungen als Terrorismus definiert werden.

Es bedarf gar nicht der von Walzer bemühten „linken Theorien über den amerikanischen Imperialismus", um diese neue Normalität mit den USA in Verbindung zu bringen, die nach 1990 als einzige Supermacht übrig geblieben sind und sich nun, was eigentlich nicht besonders überraschen sollte, mehr denn je als solche gebärdet. Der schöne Traum vom Ende der Rüstungsspirale des Kalten Krieges und von der „Friedensdividende ${ }^{s i}$ ist ausgeträumt, und die US-amerikanische Regierung selbst macht kein Geheimnis aus ihrem unilateralistischen Weltbild des "America first", das in erster Linie mit Waffengewalt durchgesetzt werden soll. So verkündete George W. Bush in seiner Rede zur "Lage der Nation" am 29. Januar 2002, oberste Priorität müsse dic „Sicherheit unserer Nation " beanspruchen, und diese sei vor allem militärisch zu gewährleisten: „Meine 
Haushaltsvorlage sieht die größte Steigerung der Verteidigungsausgaben seit zwanzig Jahren vor - weil der Preis der Freiheit und Sicherheit zwar hoch, aber nie zu hoch ist. Was immer es kostet, unser Land zu verteidigen, wir werden zahlen."

Sind diese Entwicklungen eine unmittelbare Folge des 11. September und sind dabei tatsächlich nur die USA als Hauptakteur in den Blick zu nehmen? Die Beiträge dieses Heftes zeigen, dass dies eine zu einfache Sicht der Dinge wäre. "Nichts bleibt, wie es war" ${ }^{6 \sigma}$, so verkündeten in den ersten Tagen und Wochen nach dem New Yorker Anschlag Politiker und Kommentatoren. Damals wurde von verschiedensten Seiten gefordert, Konsequenzen zu ziehen, die auf dessen Hintergründe zielen: eine stärkere Regulierung von internationalen Geldtransfers, um Geldwäsche und die Finanzierung terroristischer Netzwerke zu unterbinden; cine Neugestaltung der politischen Verhältnisse zwischen den Gewinner- und den Verlierernationen der Globalisierung, um fundamentalistischen Strömungen in ärmeren Ländern den Boden für die von ihnen geschürten Ressentiments zu entziehen; eine Unterstützung ziviler Strukturen in Regionen, die von Gewaltkulturen geprägt sind, um Alternativen zur Herrschaft von Warlords und Kriegsunternehmern zu etablieren. Fast nichts davon wurde verwirklicht, statt dessen vielmehr die Logik der militärischen Aufrüstung und des Krieges erneut in ihr Recht gesetzt. Diese verhängnisvolle politische Option ist nicht allein Sache der USA und auch keineswegs eine Neuerung der letzten Monate. Geändert haben sich durch den 11. September allenfalls die Begründungen für Waffenprogramme, sie selbst aber weisen eine Kontinuität auf, die mindestens auf die politische Wende von 1990, in manchen Fällen auch auf die Zeit davor zurückgeht. Dic Entwicklung von Kriegstechnologien, die Situation der Rüstungsindustrie, neue Militärstrategien und staatliche Politik stehen in einem komplexen Wechselverhältnis, in dem sowohl Kontinuitäten von Kriegführung als auch Brüche sichtbar werden.

So zeigt Ulrich Cremer in seinem Beitrag zur "alten" und "neuen" NATO, dass der 11. September als Katalysator für eine Reihe von militärisch-politischen Entwicklungen gewirkt hat, die sich unmittelbar nach dem Ende des Kalten Kriegs abzeichneten. Selbst das Argument der Terrorismusbekämpfung findet sich bereits Anfang der 1990er Jahre in Dokumenten des Militärbündnisses, und schon damals verabschiedete sich dieses von dem Selbstverständnis einer defensiven Organisation zugunsten des Anspruchs, weltweit überall dort einzugreifen, wo man nach eigener Einschätzung „Sicherheitsinteressen " bedroht sieht. Dies schließst nicht aus, dass es innerhalb der NATO in einzelnen Fällen, wie in den letzten Monaten etwa hinsichtlich des Iraks, unterschiedliche Einschätzungen gibt. Auch wenn dic Dominanz der US-Regierung vor allem beim Afghanistan-Krieg die anderen Teilnehmerstaaten derzeit in den Hintergrund gedrängt hat, werden die Angriffskapazitäten der NATO zielstrebig ausgebaut und gesellschaftlich kaum noch in Frage gestellt. Die eigentlichen Verlierer bei dieser NATO-Neuorientierung, so wird in diesem Artikel klar, sind die UNO und das Völkerrecht.

Sowohl Kontinuität als auch eine weitere Stufe der Eskalation zeigt sich bei den US-amerikanischen Plänen der Raketenabwehr und der Weltraumrüstung. Jürgen Scheffran analysiert die aktuelle Politik zur Aufrüstung des Weltraums, in der es nicht mehr allein um Raketenabwehr geht, sondern gleichfalls um die zukünftige Weltraumbewaffnung. Bei der Dynamik des militärischen Wettrüstens handelt es sich zu einem großen Teil um einen Wettlauf zwischen Offensive und Defensive: Angriffswaffen werden durch Abwehrmaßnahmen vorübergehend unwirksam gemacht, bis neue Offensivsy- 
steme diese wieder übertreffen. Nun findet sich auch der Weltraum, der trotz der militärischen Nutzung von Aufklärungs-, Kommunikations-, Navigationsund Wettersatelliten bislang eine waffenfreie Zone geblieben ist, von den USA in ihr neues, extrem kostspieliges und in sich widersprüchliches, Abschreckungskonzept einbezogen. Dabei laufen viele dieser offensiven Weltraumaktivitäten unter dem Deckmantel der Raketenabwehr, die damit zu einem „trojanischen Pferd" für die Weltraumrüstung wird.

Biologische Waffen gerieten für kurze Zeit in die Schlagzeilen, als Anfang Oktober in den USA Milzbrand-Anschläge verübt wurden. Als sich der Verdacht erhärtete, dass die Anschläge nicht von den dämonisierten Gegnern ausgingen, sondern aus den US-Rüstungslaboren selbst kamen, wurde es schnell wieder still um aktuelle Bedrohungen durch biologische Waffen - zu Unrecht, wie im Beitrag von Iris Hunger deutlich wird. Denn biologische Methoden der Kriegführung wurden seit dem Zweiten Weltkrieg in mehreren Staaten vorangetrieben, was schließlich dazu führte, dass in einem internationalen Übereinkommen von 1972 erstmals eine gesamte Waffengattung verboten wurde. Doch haben die bisherigen Erfahrungen gezeigt, dass die fehlenden Kontrollmechanismen insbesondere eines Verifikationssystems dessen Effektivität stark beeinträchtigen. Langjährige Bemühungen, hier Verbesserungen zu schaffen, mündeten schließlich in einen Protokollentwurf, den die USA im Juli 2001 ablehnten, womit siebenjährige diplomatische Anstrengungen mit einem Federstrich nahezu vollständig beseitigt wurden. Im selben Monat torpedierte die US-amerikanische Regierung ebenfalls die Bemühungen, auf einer UNO-Konferenz erstmalig die weltweite Verbreitung von Kleinwaffen auch nur ansatzweise kontrollierbar $\mathrm{zu}$ machen. Dennoch sind die USA in diesem Fall, wie Dorothea Schmidt darlegt, keineswegs der einzige Staat, der ein Inter- esse daran hat, den Produzenten seines Landes möglichst ungestörte Verkäufe innerhalb und außerhalb der eigenen Grenzen sowie der eigenen Regierung nach eigenem Gutdünken Waffentransfers in Krisengebiete $\mathrm{zu}$ ermöglichen. Kleinwaffen wie etwa Granaten oder $\mathrm{Ma}$ schinengewehre, die vielfach als neue Massenvernichtungswaffen bezeichnet werden, weil auf sie in den kriegerischen Konflikten des letzten Jahrzehnts der allergrößte Teil der Kriegstoten zurückgeht, wurden lange Zeit in ihrer Bedeutung unterschätzt. Ihr aktuelles Zerstörungspotential rührt nicht allein aus der Neuproduktion in einer Vielzahl von Ländem (darunter Russland, China, Israel, Deutschland und Brasilien) her, sondern auch aus Millionen von Waffen, die aus Altbeständen stammen und vor allem seit dem Ende des Kalten Krieges weltweit zirkulieren. Sie wurden in „alten $^{\text {" }}$ und werden in "neuen ${ }^{" 6}$ Kriegen eingesetzt, von staatlichen und von nicht-staatlichen Akteuren.

$\mathrm{Ob}$ es um Raketenabwehr und Weltraumflugzeuge, um biologische Kampfstoffe oder um Kleinwaffen geht - man wird die US-amerikanische Regierung derzeit immer als Hauptakteur ausmachen können, wenn es gilt, technische Entwicklungen voranzutreiben, finanzielle Mittel für derartige Zwecke umzulenken, internationale Konventionen zu verhindern oder auszuhöhlen und die Politik auf militärische Lösungen einzustimmen. Gleichwohl sollten die übrigen Akteure auf dieser Bühne nicht übersehen werden. Gerade die Bedrohung durch Atomwaffen zeigt, wie im Beitrag von Götz Neuneck ausgeführt wird, wie auch andere Staaten, insbesondere die frühere Sowjetunion, auf diese Karte setzten. Da wie dort sind die Naturwissenschaften immer wieder in einer ambivalenten Rolle zu sehen, indem sie ebenso für die Realisierung dieser Art von Kriegführung wie für deren Verhinderung eingesetzt werden konnten. Die Systemkonkurrenz der Großmächte führte 
über Jahrzehnte dazu, die Wissenschaftler im eigenen Lager neue Angriffs- und Abwehrwaffen entwickeln zu lassen. Es sind aber auch Wissenschaftler aus West und Ost, die ihre fachlichen Kompetenzen im Rahmen der Pugwash Conferences (seit 1975) und im Rahmen anderer Initiativen dazu eingesetzt haben, ein Ende der bipolaren Aufrüstung zu erreichen. Die Gefährlichkeit von Atomwaffen ist in der Gegenwart um nichts geringer geworden und wird zudem in den letzten Jahren durch technische Neuerungen ergänzt, wenn etwa Hochenergielaser oder Quantenkryptographie für militärische Zwecke nutzbar gemacht werden sollen. Insofern sind Naturwissenschaftler weiterhin dazu aufgerufen, ihr Expertenwissen zur Beobachtung solcher Entwicklungen einzusetzen, damit zumindest Beschränkungs- und Verifikationsmaßnahmen erarbeitet werden können. In diesem Zusammenhang drucken wir auch eine Erklärung der Vereinigung Deutscher Wissenschaftler (VDW) vom März 2002 ab. Die VDW sieht sich in der Tradition des RussellEinstein-Manifests von 1955 zur Ächtung von Kernwaffen und wendet sich in dieser Erklärung gegen die bekannt gewordenen Pläne der Bush-Administration zur künftigen Nuklearpolitik der USA. In einem historischen Rückblick auf die Geschichte der Industrialisierung der Kriegführung von Jens Warburg wird untersucht, wie die zunehmende Technisierung seit dem 19. Jahrhundert nicht nur dazu geführt hat, dass „leere Schlachtfelder" entstanden, sondern für die Soldaten, jedenfalls diejenigen der am weitesten entwickelten Länder, Krieg immer

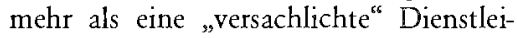
stung erscheinen musste, die nur entfernt mit dem Töten von Menschen zu tun hat. Die wachsende Entfernung des Soldaten von seinen Opfern hat frühere Formen des männlichen Heldentums abgeschafft, stellt für die obersten Kriegsherren aber dennoch eine fragile Strategie dar. Das Ziel der weiteren In- dustrialisierung liegt in der militärischen Logik daher darin, ferngesteuerte Automaten zu entwickeln, die treffsicher und ohne weitere Beeinflussung durch einen Menschen, selbständig ihr Ziel auswählen und zerstören.

Außerhalb des Schwerpunkts setzt sich Peter Marcuse mit Manuel Castells dreibändigem Werk zum Informationszeitålter auseinander, das gerade in deutscher Übersetzung erscheint. Castells Studie, die vielfach bereits als Standardwerk gilt, wird von Marcuse vor allem aufgrund einer spezifischen Tendenz zur Entpolitisierung gesellschaftlicher Konflikte kntisiert: technische Möglichkeiten und Zwänge - die man allenfalls ,gestalten" aber nicht grundsätzlich in Frage stellen kann - erscheinen als entscheidende Determinanten gesellschaftlicher Entwicklung, hinter denen unterschiedliche Interessen, sowie Macht- und Herrschaftsverhältnisse weitgehend verschwinden.

$$
\text { to * * }
$$

Das Konzept dieses Heftes haben wir gemeinsam mit Ulrich Albrecht entwikkelt, der sich aufgrund einer schweren Krankheit aber nicht mehr an seiner Realisierung beteiligen konnte. Ihm möchten wir an dieser Stelle herzlich danken. 